\section{Frequency, risk factors, and impact on mortality of arterial thromboembolism in patients with cancer}

\author{
Ella Grilz, ${ }^{1}$ Oliver Königsbrügge, ${ }^{1}$ Florian Posch,,${ }^{1,2}$ Manuela Schmidinger, ${ }^{3}$ \\ Robert Pirker, ${ }^{3}$ Irene M. Lang, ${ }^{4}$ Ingrid Pabinger ${ }^{1}$ and Cihan $\mathrm{Ay}^{1}$
}

${ }^{1}$ Clinical Division of Hematology and Hemostaseology, Department of Medicine I, Medical University of Vienna; ${ }^{2}$ Division of Oncology, Department of Internal Medicine, Medical University of Graz; ${ }^{3}$ Clinical Division of Oncology, Department of Medicine I, Medical University of Vienna and ${ }^{4}$ Clinical Division of Cardiology, Department of Medicine II, Medical University of Vienna, Austria

\section{ABSTRACT}

Tn contrast to venous thromboembolism, little is known about arterial thromboembolism in patients with cancer. The aim of this study was to quantify the risk and explore clinical risk factors of arterial thromboembolism in patients with cancer, and investigate its potential impact on mortality. Patients with newly-diagnosed cancer or progression of disease after remission were included in a prospective observational cohort study and followed for two years. Between October 2003 and October 2013, 1880 patients (54.3\% male; median age 61 years) were included. During a median follow up of 723 days, $48(2.6 \%)$ patients developed arterial thromboembolism [20 (41.7\%) myocardial infarction, $16(33.3 \%)$ stroke and $12(25.0 \%)$ peripheral arterial events], $157(8.4 \%)$ developed venous thromboembolism, and 754 (40.1\%) patients died. The cumulative 3-, 6-, 12-, and 24-month risks of arterial thromboembolism were $0.9 \%, 1.1 \%, 1.7 \%$, and $2.6 \%$, respectively. Male sex (subdistribution hazard ratio $=2.9,95 \%$ CI: 1.5-5.6; $P=0.002$ ), age (subdistribution hazard ratio per 10 year increase=1.5, 1.2-1.7; $P<0.001$ ), hypertension (3.1, 1.7-5.5; $P<0.001)$, smoking (2.0, 1.1-3.7; $P=0.022)$, lung cancer $(2.3,1.2-4.2 ; P=0.009)$, and kidney cancer $(3.8$, $1.4-10.5 ; P=0.012$ ) were associated with a higher arterial thromboembolism risk. Furthermore, the occurrence of arterial thromboembolism was associated with a 3.2-fold increased risk of all-cause mortality (hazard ratio=3.2, 95\%CI: 2.2-4.8; $P<0.001$ ). Arterial thromboembolism is a less common complication in patients with cancer than venous thromboembolism. The risk of arterial thromboembolism is high in patients with lung and kidney cancer. Patients with cancer who develop arterial thromboembolism are at a 3 -fold increased risk of mortality.

\section{Introduction}

Cancer is associated with a hypercoagulable state which leads to an increased risk of venous thromboembolism (VTE). ${ }^{1,2}$ The risk of VTE varies significantly among different cancer types and is associated with an increased risk of mortality. ${ }^{3,4}$ In contrast to VTE, much less is known on the epidemiology of arterial thromboembolism (ATE) in patients with cancer. . $^{5-7}$

It is important to identify an association between ATE and cancer because cardiovascular diseases and cancer are becoming more prevalent in an aging population and may share common risk factors and pathobiology related to inflammation. ${ }^{8,9}$ Furthermore, recent advances in screening, diagnosis and therapy have improved the survival of many cancers, implying that the population of patients with cancer now lives longer and develops a risk for cardiovascular complications. ${ }^{10,11}$ The risk of ATE in cancer may also be associated with anti-neoplastic treatments, known for the risk of arterial complications. ${ }^{12}$

As data on the epidemiology, risk and burden of ATE in patients with cancer are
Haematologica 2018

Volume 103(9):1549-1556

\section{Correspondence:}

cihan.ay@meduniwien.ac.at

Received: March 1, 2018.

Accepted: May 17, 2018.

Pre-published: May 24, 2018.

doi:10.3324/haematol.2018.192419

Check the online version for the most updated information on this article, online supplements, and information on authorship \& disclosures: www.haematologica.org/content/103/9/1549

(C)2018 Ferrata Storti Foundation

Material published in Haematologica is covered by copyright. All rights are reserved to the Ferrata Storti Foundation. Use of published material is allowed under the following terms and conditions:

https://creativecommons.org/licenses/by-nc/4.0/legalcode. Copies of published material are allowed for personal or internal use. Sharing published material for non-commercial purposes is subject to the following conditions:

https://creativecommons. org//icenses/by-nc/4.0/legalcode, sect. 3. Reproducing and sharing published material for commercial purposes is not allowed without permission in writing from the publisher. 
scarce, we conducted a study to describe the incidence of ATE, explore clinical risk factors, and investigate the impact of ATE on mortality in patients with cancer.

\section{Methods}

\section{Study design}

This study was performed within the framework of the Vienna Cancer and Thrombosis Study (CATS), which started in 2003 at the Medical University of Vienna. CATS is a single-center prospective observational cohort study, approved by the ethics committee (number: 126/2003, ethik-kom@meduniwien.ac.at), and conducted in accordance with the Declaration of Helsinki. Detailed information about the study design and procedures have been reported previously. ${ }^{13}$ Briefly, adult patients ( $\geq 18$ years) with a newly diagnosed malignancy or progression of disease after complete or partial remission were eligible for inclusion. Patients were not included if they had received radiotherapy or surgery within the last two weeks or chemotherapy within the last three months before study inclusion. Furthermore, all patients with a thromboembolic event within the last three months or an overt bacterial or viral infection within the last six weeks before study inclusion were excluded. Patients with an indication for long-term prophylactic or therapeutic anticoagulation were excluded, but temporary treatment with low molecular heparin (e.g. for hospitalized patients) was allowed. Furthermore, patients on acetylsalicylic acid or other platelet inhibitors were not excluded. ${ }^{13,14}$ All patients gave their written informed consent and were prospectively followed for a maximum duration of two years, until the occurrence of VTE, loss of follow up, withdrawal of consent, or death.

Until October 2013, 2004 patients were included in this study. After re-evaluation of the inclusion and exclusion criteria, 124 patients had to be excluded, because: 1) they did not fulfill inclusion $(n=35)$ or exclusion criteria $(n=60) ; 2)$ no follow up was available $(\mathrm{n}=20)$; 3) no material for laboratory analyses was available $(n=7)$; or 4) patients withdrew consent $(n=2)$. Thus, overall 1880 patients with active cancer between $17^{\text {th }}$ October 2003 and $28^{\text {th }}$ October 2013 were included in this analysis.

\section{Outcome measurement}

Venous thromboembolism is the pre-defined primary outcome of CATS. Data on ATE were collected as a comorbid condition during the observation time. To verify diagnosis of ATE, we compiled data from: 1) the CATS database; 2) patients' follow-up letters; 3) telephone records with patients, their family members, treating oncologists/hemato-oncologists and general practitioners during follow up; 4) digital information systems of the General Hospital of Vienna and the Vienna Urban Health Care Providers; and 5) the Austrian death registry.

The primary end point of this analysis was objectively confirmed symptomatic ATE, which was defined as a composite of acute myocardial infarction (ST-elevation myocardial infarction and non-ST-elevation myocardial infarction), peripheral arterial occlusion, if treated with an interventional procedure (i.e. a catheter-based or open surgical procedure to improve arterial blood flow in non-cardiac arteries, except the intracranial vessels), and ischemic stroke. Both minor (National Institute of Health Stroke Score $\leq 3$ ) and major (National Institute of Health Stroke Score > 3) stroke were included. ${ }^{15,16} \mathrm{~A}$ panel of experts in cardiology, neurology, and vascular medicine adjudicated all events based on objective evidence. Objective evidence for diagnosis included: 1) computed tomography (CT); magnetic resonance imaging (MRI) and autopsy report for ischemic stroke; 2) Doppler-sonography, digital subtraction angiography, CT-angiography, and MR- angiography for peripheral arterial occlusion; 3) electrocardiography, echocardiography (e.g. hypokinetic/akinetic and hypotrophic myocardial section without any other existing reason), cardiac biomarkers, identification of an intracoronary thrombus by angiography, and autopsy evidence for myocardial infarction. No routine screening for ATE was carried out during the study. Asymptomatic arterial thrombosis (e.g. incidentally detected stroke on restaging CT scans) was considered an event if it was considered clinically significant by members of the adjudication committee. In cases where objective diagnostic evidence based on imaging or lab results was missing, the adjudication committee decided on the basis of documented clinical evidence. The adjudication committee also classified mortality into fatal ATE and death-from-any-cause-other-than-fatal-ATE, which was the secondary end point.

\section{Statistical analysis}

Continuous variables were summarized as medians $\left(25^{\text {th }}-75^{\text {th }}\right.$ percentile), and count data as absolute frequencies (\%). The reverse Kaplan-Meier method was used to estimate median follow-up time. ${ }^{17}$

The cumulative incidence of ATE was calculated using a competing risk estimator with $95 \%$ confidence intervals $(95 \% \mathrm{CI}){ }^{18}$ ATE incidences between groups were compared with Gray's tests. ${ }^{19}$ We assume that cancer patients are not only at risk of ATE, but are also at risk of dying from cancer. If patients die from their underlying malignancy, the risk of ATE occurrence is instantly reduced to zero. Therefore, death represents a competing risk scenario. ${ }^{20,21}$ To address this issue, univariable and multivariable Fine \& Gray competing risk regression models were used to analyze the subdistribution hazards of ATE. ${ }^{22}$ Overall survival was analyzed with Kaplan-Meier estimators, and hazards of death were modeled with uni- and multivariable Cox models. VTE and deathfrom-any-cause-other-than-fatal-ATE were considered competing events in all statistical analyses concerning the primary end point. ${ }^{20}$ Relative risks of ATE between patients with breast cancer and other tumor types could not be estimated because the risk of ATE was $0.0 \%$ in breast cancer patients. Thus, modeling for this comparison was performed with a generalized linear model from the Bernoulli family with an identity link. ${ }^{23}$

To quantify the impact of ATE occurring during follow up on mortality, we used Cox models treating ATE as a time-dependent variable (controlling for immortal time bias), and performed a landmark analysis. In the time-dependent Cox model, one day of survival time was added in one patient who developed ATE and died on the same day.

Stata 14.0 (Stata Corp., Houston, TX, USA) and SPSS 24 (SPSS Inc., Chicago IL, USA) were used to perform all statistical analyses.

\section{Results}

\section{Patients' and follow-up characteristics}

The study cohort included 1880 patients with a wide range of different cancer types (Table 1). The majority of patients $(n=1385,73.7 \%$ ) had newly diagnosed cancer, while 495 (26.3\%) patients had a progressive disease after complete or partial remission. The median follow-up time was 723 days $\left[25^{\text {th }}-75^{\text {th }}\right.$ percentile (Q1-O3): $308-731$, range: 1-731]. During the observation time 157 (8.4\%) VTE events were observed and 754 (40.1\%) patients died.

\section{Risk of ATE in patients with cancer}

Forty-eight (2.6\%) patients developed ATE during the observation time. Among those patients, 19 (39.6\%) non- 
fatal myocardial infarctions, $16(33.3 \%)$ ischemic strokes, $12(25.0 \%)$ peripheral arterial events, and one (2.1\%) fatal myocardial infarction were observed. Detailed information on cancer patients with ATE is listed in Table 2. The cumulative 3-, 6-, 12-, and 24-month ATE risks were $0.9 \%$ (95\% CI: 0.6-1.4), 1.1\% (0.7-1.7), 1.7\% (1.2-2.4), and 2.6\% (2.0-3.4), respectively (Figure 1). While the rate of VTE was highest during the first six months of follow up and strongly declined thereafter, ATE events did not have a peak incidence, but rather occurred at a relatively constant rate (Figure 2).

Table 1. Baseline characteristics of the total study population.

\begin{tabular}{lcc} 
& N. of patients & $\%$ \\
Median age at study entry, years & 61 & \\
First to third quartile & $52-68$ & \\
\hline Median body mass index, $\mathrm{kg} / \mathrm{m}^{2}$ & 25.1 & \\
First to third quartile & $22.4-28.3$ & \\
Sex & & \\
Female & 860 & 45.7 \\
Male & 1020 & 54.3 \\
Site of cancer & & \\
Lung & 319 & 17.0 \\
Breast & 276 & 14.7 \\
Lymphoma & 265 & 14.1 \\
Brain & 248 & 13.2 \\
Colorectal & 186 & 9.9 \\
Prostate & 157 & 8.4 \\
Pancreas & 133 & 7.1 \\
Stomach & 65 & 3.5 \\
Multiple myeloma & 50 & 2.7 \\
Kidney & 45 & 2.4 \\
Others & 136 & 7.2 \\
\hline Progresion & & \\
\end{tabular}

Progression of tumor

\begin{tabular}{lcc} 
Localized & 629 & 35.7 \\
Distant metastasis & 627 & 35.6 \\
Not classifiable $^{\mathrm{a}}$ & 506 & 28.7 \\
Smoking status & & \\
Smoker & 546 & 31.6 \\
Ex-smoker (>1-year non-smoker) & 313 & 18.1 \\
Non-smoker & 867 & 50.2 \\
\hline Hypertension at study entry & 704 & 37.5 \\
Diabetes at study entry & 223 & 11.9 \\
Known atherosclerotic cardiovascular & 159 & 8.5 \\
disease at study entry & & \\
Dyslipidemia at study entry & 222 & 11.9 \\
\hline History of venous thromboembolism* & 91 & 4.8 \\
Platelet aggregation inhibitor use & 280 & 14.9 \\
at study entry & & \\
\hline
\end{tabular}

\begin{tabular}{lll}
\hline Lipid lowering agent use at study entry $\quad 238$ & 12.7
\end{tabular}

Continuous data are reported as medians with first and third quartiles. Categorical variables are given as absolute frequencies and percentages. ${ }^{2}$ Data on body mass index, progression of tumor, and smoking status are missing in 6,118, and 154 patients, respectively. *Defined as venous thromboembolism (VTE) that had occurred more than three months before study inclusion. N: number.

\section{Risk factors for ATE in patients with cancer}

In univariable competing risk regression analysis, male sex [subdistribution hazard ratio (SHR) $=2.9,95 \% \mathrm{CI}: 1.5$ 5.6; $P=0.002]$, higher age (SHR per 10 year increase $=1.5$, 1.2-1.7; $P<0.001)$, hypertension (SHR=3.1, 1.7-5.5; $P<0.001)$, diabetes $(\mathrm{SHR}=2.2,1.2-4.4 ; P=0.020)$, a positive

Table 2. Baseline characteristics of patients with ATE.

\begin{tabular}{lcc} 
& N. of patients & $\%$ \\
Median age at study entry, years & 66 & \\
First to third quartile & $60-69$ & \\
\hline Median body mass index, $\mathrm{kg} / \mathrm{m}^{2}$ & 26.3 & \\
First to third quartile & $23.2-29.0$ & \\
\hline Sex & & \\
Female & 11 & 22.9 \\
Male & 37 & 77.1 \\
Type of arterial thromboembolic event & & \\
Myocardial infarction & 20 & 41.7 \\
Major stroke & 13 & 27.1 \\
Minor stroke & 3 & 6.3 \\
Peripheral arterial occlusion & 12 & 25.0 \\
\hline
\end{tabular}

Site of cancer

\begin{tabular}{lcc} 
Lung & 15 & 45.8 \\
Breast & 0 & 0.0 \\
Lymphoma & 5 & 10.4 \\
Brain & 7 & 14.6 \\
Colorectal & 3 & 6.3 \\
Prostate & 8 & 16.7 \\
Pancreas & 2 & 4.2 \\
Stomach & 2 & 4.2 \\
Multiple myeloma & 0 & 0.0 \\
Kidney & 4 & 8.4 \\
Others & 2 & 4.2 \\
Progression of tumor & & \\
Localized & 19 & 44.2 \\
Distant metastasis & 14 & 32.6 \\
Not classifiable & 10 & 23.3 \\
\hline Smoking status & & \\
Smoker & 19 & 40.4 \\
Ex-smoker (>l-year non-smoker) & 12 & 25.5 \\
Non-smoker & 16 & 34.0 \\
Hypertension at study entry & 31 & 64.6 \\
\hline Diabetes at study entry & 11 & 22.9 \\
Known atherosclerotic cardiovascular & 12 & 25.0 \\
disease at study entry & & \\
Dyslipidemia at study entry & 8 & 16.7 \\
History of venous thromboembolism* & 2 & 4.2 \\
\hline Platelet aggregation inhibitor use & 22 & 45.8 \\
at study entry & & \\
Lipid lowering agent use at study entry & 14 & 29.2 \\
\hline Cotn & & \\
\hline & & \\
\hline
\end{tabular}

Continuous data are reported as medians with first and third quartiles. Categorical variables are given as absolute frequencies and percentages. a Data on progression of tumor are missing in 5 patients. Data on smoking status are missing in one patient. *Defined as venous thromboembolism (VTE) that had occurred more than three months before study inclusion. N: number. 
smoking history $(\mathrm{SHR}=2.0,1.1-3.7 ; P=0.022)$, and a known arterial cardiovascular disease (e.g. history of stroke, peripheral arterial disease, coronary heart disease) at study entry $(\mathrm{SHR}=3.7,1.9-7.2 ; P<0.001)$ were associated with a higher ATE risk.

The body mass index $(\mathrm{SHR}=1.0,1.0-1.1 ; P=0.115)$, and a prior history of VTE (SHR=0.8, 0.2-3.5; $P=0.814)$ were not associated with risk of ATE. Furthermore, dyslipidemia, which was defined as having at least one of the following diagnoses: 1) hypertriglyceridemia; 2) hyperlipidemia; or 3) hypercholesterolemia, was also not associated with the risk of ATE in patients with cancer $(\mathrm{SHR}=1.5$, 0.7-3.2; $P=0.302)$. Treatment with lipid lowering agents $(\mathrm{SHR}=2.9,1.5-5.3 ; P<0.001)$ or platelet aggregation inhibitors $(\mathrm{SHR}=5.0,2.8-8.8 ; P<0.001)$ at study entry was associated with ATE occurrence. Cancer stage was not associated with an increased risk of ATE occurrence (SHR=0.7, 0.4-1.5; $P=0.398)$. Lung cancer $(\mathrm{SHR}=2.3$, 1.2$4.2 ; P=0.009)$, and kidney cancer $(\mathrm{SHR}=3.8,1.4-10.5$; $P=0.012)$ were associated with an increased risk of ATE. Cumulative incidences of ATE separated by cancer type are shown in Figure 3. In multivariable competing risk regression analyses, age [adjusted (adj.) SHR per 10 year increase $=1.4$, 95\%CI: 1.2-1.7; $P<0.001]$, male sex (adj. $\mathrm{SHR}=2.6,1.3-5.2 ; P=0.006$ ), and smoking (adj. $\mathrm{SHR}=2.1$, 1.1-3.9; $P=0.026$ ) emerged as independently associated with the risk of ATE in patients with cancer when corrected for each other. After correction for age, male sex, and smoking, the association between hypertension (adj. $\mathrm{SHR}=2.4$, 1.3-4.5; $P=0.005$ ), a known arterial cardiovascular disease (adj. SHR=2.6, 1.3-5.3; $P=0.007$ ), treatment with lipid lowering agents (adj. $\mathrm{SHR}=2.2$, 1.2-4.4; $P=0.013$ ), and the use of platelet aggregation inhibitors (adj. SHR=3.7, 2.1-6.7; $P<0.001$ ) at study entry and ATE remained statistically significant. Diabetes did not reach statistical significance in this multivariable analysis (adj. $\mathrm{SHR}=1.8,0.9-3.5 ; P=0.093)$. Furthermore, the association between ATE and kidney cancer prevailed (adj. SHR=3.7, 1.3-10.6; $P=0.016$ ), whereas it did not for lung cancer (adj.
SHR=1.6, 0.8-3.4; $P=0.193)$. None of the 276 patients with breast cancer developed ATE during follow up (crude risk $=0.0 \%$ ). In contrast, 48 ATE events occurred in the 1604 patients with other tumor entities (crude risk=3.0\%), for an absolute risk difference of 3.0\% (95\% CI: 1.0-5.0; $P=0.004)$. However, breast cancer patients were significantly younger than patients with other tumors (median age 59 vs. 62 years; $P<0.001$ ). Nonetheless, breast cancer remained associated with a lower risk of ATE even after adjusting for age (adjusted absolute risk difference $=2.8 \%$, 95\% CI: $0.7-4 ; \quad P=0.007$ ), and also after exclusion of male patients and adjustment for age (adjusted absolute risk difference $=1.7 \%$, 95\% CI: $0.1-3.3 ; P=0.035$ ).

We also investigated the cumulative impact of cardiovascular risk factors on ATE risk in patients with cancer (Figure 4). We assigned one point for each of the following cardiovascular risk factors: hypertension, diabetes, known arterial cardiovascular disease, and dyslipidemia. Assuming a linear relationship between the number of risk factors and ATE risk, the SHR was 1.8 (1.4-2.2; $P<0.001)$ per point increase. This association prevailed after adjustment for age and sex $(\mathrm{SHR}=1.6,1.2-2.0$; $P=0.001)$. The 2 -year cumulative incidence of ATE in patients with $0,1,2,3$, and 4 points was $1.4 \%(0.8-2.3)$, $2.7 \%(1.5-4.3), 5.8 \%(3.3-9.3), 5.6 \%(1.8-12.7)$, and $12.5 \%$ (2.1-32.8), respectively.

\section{Association between ATE and survival of patients with cancer}

The 3-, 6-, 12-, and 24-month overall survival estimates of the study cohort were $94.0 \%$ (95\%CI: 92.8-95.0), $87.5 \%(85.9-88.9), 74.8 \%(72.7-76.7)$, and $57.9 \%(55.5-$ $60.2)$, respectively. In multistate modeling the occurrence of ATE was associated with a 3.2-fold relative increase in the risk of death from any cause [hazard ratio $(\mathrm{HR})=3.2$, 95\% CI: 2.2-4.8; $P<0.001)$. This association prevailed after adjusting for age (adj. HR=2.9, 2.0-4; $P<0.001$ ), as well as age and lung cancer as an indicator for a cancer type with poor prognosis (adj. $\mathrm{HR}=2.5$, 1.7-3.7; $P<0.001$ ). In a land-

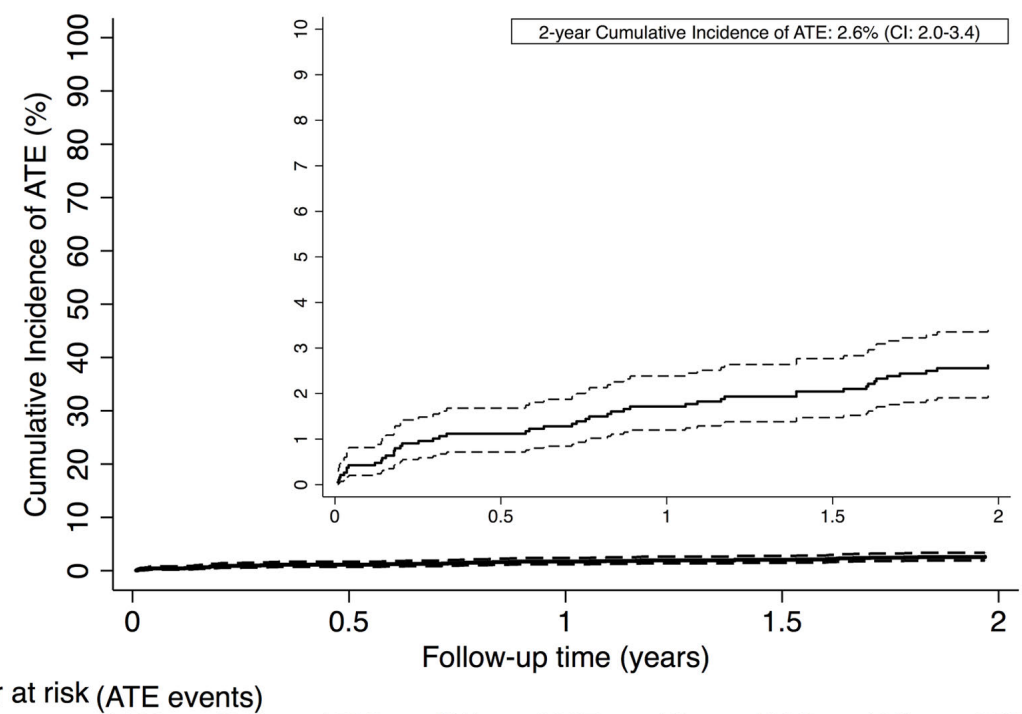
$1880 \quad(21)$
$1588 \quad(11)$

1307

(6)
1098

(10)
879
Figure 1. Cumulative incidence of arterial thromboembolism (ATE) in patients with cancer. For estimation we used a competing risk cumulative incidence estimator. Venous thromboembolism (VTE) and death-from-any-cause were considered as competing risk events. The dashed line represents 95\% confidence bands. 
mark analysis with the landmark set at three months after baseline, 2-year predicted overall survival was $62.3 \%$ in patients who did not develop ATE during the first three months of follow up, and $24.8 \%$ in patients who did develop ATE during the first three months (Mantel-Byar $P<0.001)$ (Figure 5). The median survival time of patients with cancer after ATE was only 63 days (Q1-Q3: 36-233).

\section{Discussion}

We analyzed the cumulative incidence of ATE, identified clinical risk factors, and evaluated the impact of ATE on mortality in patients with cancer in this prospective observational cohort study. During a follow-up period of up to two years, $2.6 \%$ of patients developed ATE, defined as the composite of myocardial infarction, stroke and peripheral artery disease, which was less common than VTE. The risk of ATE was increased in patients with higher age, male sex, hypertension, and a positive smoking history. In addition, the risk of ATE varied by cancer type with lung and kidney cancer having the highest, and breast cancer having the lowest risk. We also observed that the occurrence of ATE is associated with a 3-fold increased risk of mortality. The negative impact of ATE on cancer patients' prognosis indicates an unmet need for better understanding of the burden of ATE in cancer, the identification of patients at risk of ATE, and improved strategies to prevent, treat and manage ATE in patients with cancer.

An increased risk of stroke and coronary heart disease in patients with cancer has been reported in previous studies. $^{24-30}$ In a recent retrospective analysis of a Surveillance, Epidemiology, and End Results (SEER) and Medicare linked dataset of patients with a primary cancer diagnosis between 2002 and 2011 in the USA, the 6month cumulative incidence of myocardial infarction and ischemic stroke was $4.7 \%$ compared to $2.2 \%$ in a

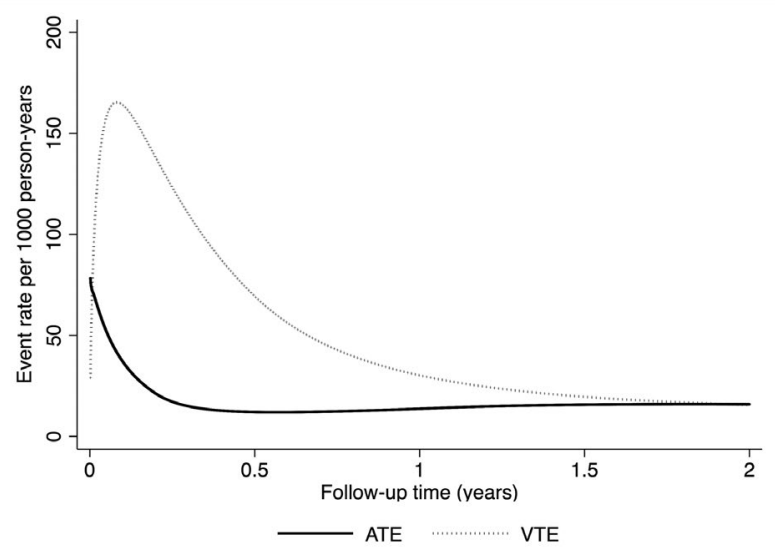

Figure 2. Time-dependent rates of arterial thromboembolism (ATE) and venous thromboembolism (VTE) over two years of follow up. While the rate of VTE was highest during the first six months of follow up and then declined, the risk of ATE did not have a peak and remained relatively constant during follow up. The curves were predicted with a flexible parametric regression model on the logcumulative-hazard scale (Stata routine stpm2). matched control group without cancer. ${ }^{31}$ The cumulative incidence of ATE in this study was higher than in our study $(4.7 \%$ vs. $2.6 \%)$. However, increasing age is an important cardiovascular risk factor and patients in this retrospective study were older than in our study, where outcome data were prospectively collected (median age 74 vs. 61 years). It is likely that an increased rate of ATE may be attributable to some degree to differences in age and also to the presence of classical cardiovascular risk factors in the studied populations. In general, estimating the true risk of ATE may be challenging in cancer patients. Nonspecific ATE symptoms such as dyspnea and chest pain are highly prevalent in cancer patients and oncologists may attribute such symptoms often to the underlying cancer, which may lead to an under-diagnosis of ATE. On the other hand, cardiologists may be reluctant to schedule cancer patients for diagnostic coronary angiographies when cancer patients have a poor performance status or are deemed to have a poor prognosis, leading to a further underestimation of the ATE burden in cancer patients. Thus, oncologists and cardiologists should maintain a relatively high index of suspicion for ATE when dealing with cancer patients with ATE-related symptoms.

The relative risk of ATE according to the cancer type in our study was similar to that of previous studies. Patients with lung and kidney cancer had the highest relative risk of ATE. ${ }^{29-31}$ The difference in ATE risk between cancer types may be driven by general risk factors that increase risk of both cancer and ATE, such as smoking, or by specific anti-cancer treatments, such as platinum-based combination chemotherapy, vascular endothelial growth factor (VEGF) / vascular endothelial growth factor receptor (VEGFR) inhibitors, which have been associated with increased risk of ATE. ${ }^{12,32-37}$ Prior studies also suggested an association between metastasis and the risk of ATE occurrence, which was not confirmed in our study. ${ }^{29-31}$

The risk of VTE in patients with cancer varies during the course of the cancer disease. It is highest during the first

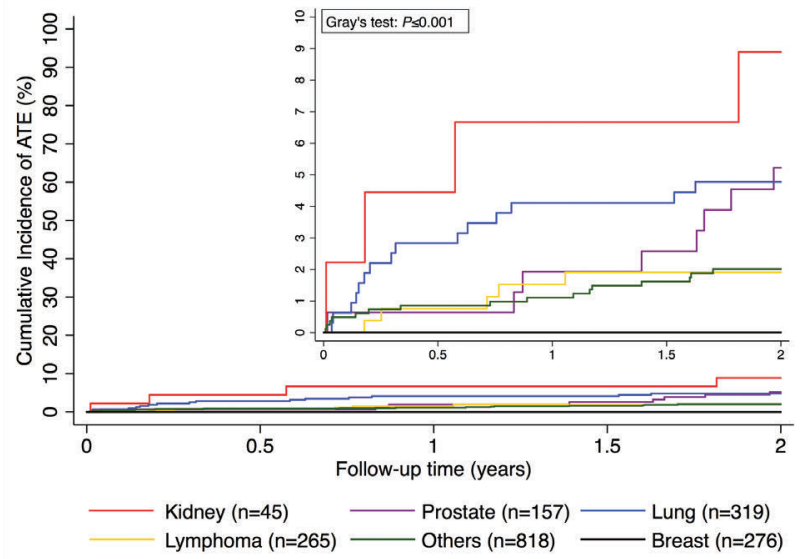

Figure 3. Cumulative incidence of arterial thromboembolism (ATE) according to cancer type. A competing risk cumulative incidence estimator was used. For clarity, only selected tumor entities are compared. A Gray's test was used to test for differences between cancer types $(P<0.001)$, venous thromboembolism and death-from-any-cause were considered as competing risk event. 
six months after diagnosis of cancer and then declines. ${ }^{38}$ Using flexible parametric modeling, we could demonstrate that in contrast to VTE, the rate of ATE does not appear to have a peak but remained relatively constant over the whole follow-up period. Hence, long-term strategies to effectively prevent ATE in cancer patients, and especially in cancer survivors, are needed. Further research has to be conducted to investigate the optimal management strategies. In this regard, a phase I trial is currently underway evaluating aspirin and statin in patients with cancer to prevent thrombosis (clinicaltrials.gov identifier: 02285738). ${ }^{39}$ We can speculate whether a primary antithrombotic prevention of ATE would have the greatest net-clinical-benefit in tumor entities with a high absolute ATE risk, such as in patients with lung and renal cancer, or in patients with individual risk factors for ATE.

The link between cancer and ATE could be explained in part by common risk factors, such as higher age and smoking. However, it is unlikely that the ATE burden in cancer patients is completely attributable to these and other general risk factors. In our study, only hypertension and a known arteriosclerotic cardiovascular disease were independently associated with the risk of ATE. We also examined the association of other cardiovascular risk factors and the occurrence of ATE in patients with cancer. Neither the body mass index nor diabetes, dyslipidemia, or prior VTE at study entry were independently associated with the risk of ATE. However, these analyses might be under-powered due to the low absolute number of patients with ATE. In contrast, the use of lipid lowering
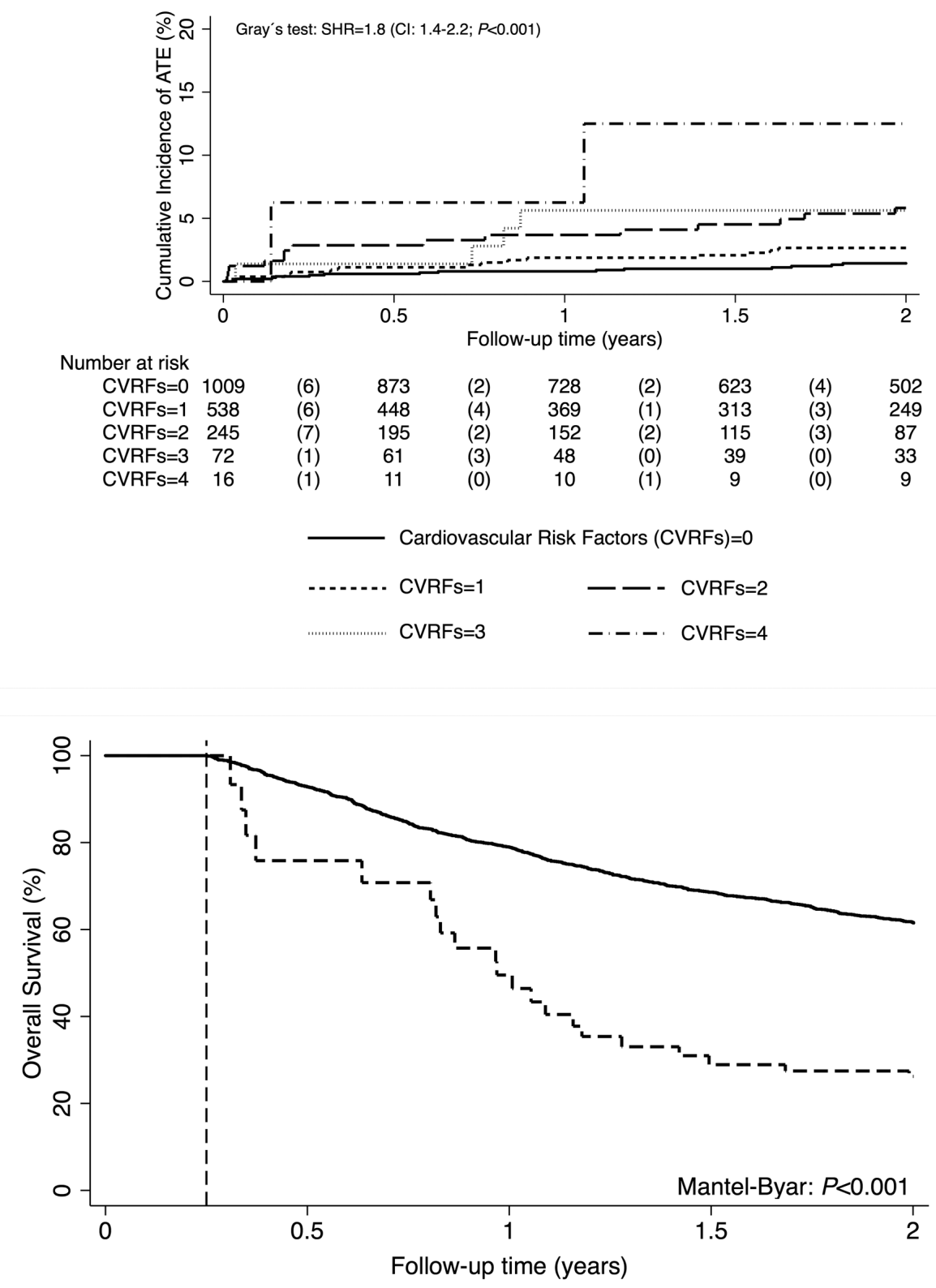

No ATE within first 3 months
---- ATE within first 3 months
Figure 4. Cumulative impact of cardiovascular risk factors on the risk of arterial thromboembolism (ATE). Competing risk analysis was used to analyze the incidence of ATE, considering venous thromboembolism (VTE) and death-from-anycause as competing risk events. SHR: subdistribution hazard ratio;CVRF: cardiovascular risk factors.

Figure 5. Landmark analysis of predicted overall survival according to arterial thromboembolism (ATE) status after three months of follow up. 1745 patients survived for at least three months ("landmark time"), of whom 13 had developed ATE within the first three months and had survived until the landmark time. Four patients had developed ATE within the first three months and died before the landmark time; 31 patients had developed ATE after the landmark time. "Predicted" instead of "observed" overall survival was chosen due to the small numbers of patients who had developed ATE within the first three months $(n=13)$. 
agents and platelet aggregation inhibitors were associated with ATE risk. However, previous studies show that a high pill burden, co-morbidities, and provision of care by multiple physicians compromise medication adherence; ${ }^{40,41}$ we assume that all of these are likely to be of relevance in patients with cancer. Therefore, we cannot exclude a bias in this analysis due to a possible lack of medication adherence or persistence. Another possible explanation for the association of use of lipid lowering drugs and platelet aggregation inhibitors could be that physicians had correctly identified those patients with a high cardiovascular risk, and consequently had prescribed this medication.

Also cancer-specific risk factors such as anti-cancer treatments (e.g. radiotherapy, platinum-containing chemotherapy, treatment with monoclonal antibodies, tyrosine kinase inhibitors) are known to increase the risk of ATE. ${ }^{24,34-36,42}$ The major limitation of our study is that we were not able to specifically model the time-dependent contribution of selected cancer therapies, such as platinum and VEGF-targeted agents, to risk of ATE, because not all information on treatment regimens was available. Furthermore, patients receiving therapeutic or prophylactic anticoagulation were excluded from the study, which might mean that this study population is less representative.

Another main finding of our study was that the occurrence of ATE during follow up is associated with an increased risk of mortality. Patients who developed ATE had a 3-fold higher risk of mortality. This is consistent with a recent study that reported a 4-fold increased risk of mortality in patients with cancer and ATE. ${ }^{31}$ Collectively, these results demonstrate that incidental ATE is a major contributor to death in patients with cancer, and that special medical attention is needed for patients with cancer and arterial thromboembolic complications to improve their prognosis. With regard to fatal ATE, we have to mention that the rate of fatal ATE in our study cohort is lower than in non-cancer patients. ${ }^{43,44}$ Therefore, we cannot exclude a possible reporting bias because no routine autopsies were performed in patients included in CATS and it is likely that physicians generally tend to attribute death to the underlying malignancy.

Although previous studies had a larger sample size, these data are taken from retrospective analyses, selected cancer entities or from post-hoc analyses of studies that were not designed to investigate ATE in cancer. ${ }^{45-48}$ To our knowledge, this is the first study in which data on ATE in cancer patients were collected prospectively. To minimize any potential bias, we retrospectively evaluated different sources for ascertainment of the ATE outcome data and a committee adjudicated all events.

In conclusion, this study used subject-level data of a well-characterized cancer cohort to define the epidemiology of ATE in patients with malignant diseases. ATE in patients with cancer is a less frequent complication than VTE. In certain cancer types, such as lung and kidney cancer, the risk of ATE is still as high as the risk of VTE. Furthermore, occurrence of ATE in patients with cancer is associated with an increased risk of mortality, calling for future clinical research efforts to better characterize patients with cancer at risk of ATE and reduce the burden of arterial thromboembolic complications.

\section{Acknowledgments}

We thank the members of the adjudication committee: Thomas Gremmel (Clinical Division of Angiology, Department of Medicine II, Medical University of Vienna) and Fritz Leutmezer (Department of Neurology, Medical University of Vienna).

\section{Funding}

The study was funded by the Anniversary Fund of the Austrian National Bank ("Jubiläumsfonds der Österreichischen Nationalbank (OeNB)" project numbers \#10935, \#12739 and \#14744) and the Austrian Science Fund (FWF), Special Research Program (SFB) 54.

\section{References}

1. Ay C, Pabinger I. Predictive potential of haemostatic biomarkers for venous thromboembolism in cancer patients. Thromb Res. 2012;129 Suppl 1:S6-9.

2. Riedl J, Pabinger I, Ay C. Platelets in cancer and thrombosis. Hamostaseologie. 2014;34(1):54-62.

3. Pabinger I, Thaler J, Ay C. Biomarkers for prediction of venous thromboembolism in cancer. Blood. 2013;122(12):2011-2018.

4. Königsbrügge $\bigcirc$, Pabinger I, Ay C. Risk factors for venous thromboembolism in cancer: novel findings from the Vienna Cancer and Thrombosis Study (CATS). Thromb Res. 2014;133 Suppl 2:S39-43.

5. Di Nisio M, Ferrante N, Feragalli B, et al. Arterial thrombosis in ambulatory cancer patients treated with chemotherapy. Thromb Res. 2011;127(4):382-383.

6. El Sakka K, Gambhir RPS, Halawa M, Chong P, Rashid H. Association of malignant disease with critical leg ischaemia. $\mathrm{Br}$ J Surg. 2005;92(12):1498-1501.

7. Javid M, Magee TR, Galland RB. Arterial
Thrombosis Associated with Malignant Disease. Eur J Vasc Endovasc Surg. 2008; 35(1):84-87

8. Prisco D, D'Elios MM, Cenci C, Ciucciarelli L, Tamburini C. Cardiovascular oncology: a new discipline inside internal medicine? Intern Emerg Med. 2014;9(4):359-364.

9. Abe J, Martin JF, Yeh ETH. The Future of Onco-Cardiology. Circ Res. 2016; 119(8):896-899.

10. Vasu S, Hundley WG. Understanding cardiovascular injury after treatment for cancer: an overview of current uses and future directions of cardiovascular magnetic resonance. J Cardiovasc Magn Reson. 2013; 15:66.

11. DeSantis CE, Lin CC, Mariotto AB, et al. Cancer treatment and survivorship statistics, 2014. CA Cancer J Clin. 2014; 64(4):252-271.

12. Choueiri TK, Schutz FAB, Je Y, Rosenberg JE, Bellmunt J. Risk of Arterial Thromboembolic Events With Sunitinib and Sorafenib: A Systematic Review and Meta-Analysis of Clinical Trials. J Clin Oncol. 2010;28(13):2280-2285.

13. Ay C, Simanek R, Vormittag R, et al. High plasma levels of soluble P-selectin are pre- dictive of venous thromboembolism in cancer patients: results from the Vienna Cancer and Thrombosis Study (CATS). Blood. 2008;112(7):2703-2708.

14. Ay C, Vormittag R, Dunkler D, et al. Ddimer and prothrombin fragment $1+2$ predict venous thromboembolism in patients with cancer: results from the Vienna Cancer and Thrombosis Study. J Clin Oncol. 2009;27(25):4124-4129.

15. Thygesen K, Alpert JS, Jaffe AS, et al. Third universal definition of myocardial infarction. Eur Heart J. 2012;33(20):2551-2567.

16. Fischer U, Baumgartner A, Arnold M, et al What Is a Minor Stroke? Stroke. 2010;41(4):661-666.

17. Schemper M, Smith TL. A note on quantifying follow-up in studies of failure time. Control Clin Trials. 1996;17(4):343-346.

18. Coviello V, Boggess M. Cumulative incidence estimation in the presence of competing risks. Stata J. 2004;4(2):103-112.

19. Gray RJ. A Class of K-Sample Tests for Comparing the Cumulative Incidence of a Competing Risk. Ann Stat. 1988; 16(3):1141-1154.

20. Ay C, Posch F, Kaider A, Zielinski C, Pabinger I. Estimating risk of venous 
thromboembolism in patients with cancer in the presence of competing mortality. J Thromb Haemost. 2015;13(3):390-397.

21. Delgado J, Pereira A, Villamor N, LopezGuillermo A, Rozman C. Survival analysis in hematologic malignancies: recommendations for clinicians. Haematologica. 2014;99(9):1410-1420.

22. Fine JP, Gray RJ. A Proportional Hazards Model for the Subdistribution of a Competing Risk. J Am Stat Assoc. 1999; 94(446):496-509.

23. Terbuch A, Posch F, Annerer LM, et al. Long-term cardiovascular complications in stage I seminoma patients. Clin Transl Oncol. 2017;19(11):1400-1408.

24. McGale P, Darby SC, Hall P, et al. Incidence of heart disease in 35,000 women treated with radiotherapy for breast cancer in Denmark and Sweden. Radiother Oncol. 2011;100(2):167-175

25. Chen PC, Muo CH, Lee YT, Yu YH, Sung FC. Lung cancer and incidence of stroke: a population-based cohort study. Stroke. 2011:42(11):3034-3039.

26. Kuan AS, Teng CJ, Wu HH, et al. Risk of ischemic stroke in patients with ovarian cancer: a nationwide population-based study. BMC Med. 2014;12(1):53.

27. Kuan AS, Chen SC, Yeh CM, et al. Risk of Ischemic Stroke in Patients With Gastric Cancer. Medicine (Baltimore). 2015; 94(37):e1336.

28. Navi BB, Reiner AS, Kamel $\mathrm{H}$, et al Association between incident cancer and subsequent stroke. Ann Neurol 2015; 77(2):291-300.

29. Zöller B, Ji J, Sundquist J, Sundquist K. Risk of coronary heart disease in patients with cancer: A nationwide follow-up study from Sweden. Eur J Cancer. 2012;48(1):121-128.

30. Zöller B, Ji J, Sundquist J, Sundquist K. Risk of haemorrhagic and ischaemic stroke in patients with cancer: A nationwide followup study from Sweden. Eur J Cancer. 2012;48(12):1875-1883.
31. Navi BB, Reiner AS, Kamel H, et al. Risk of Arterial Thromboembolism in Patients With Cancer. J Am Coll Cardiol. 2017;70(8):926-938.

32. Ranpura V, Hapani S, Chuang J, Wu S. Risk of cardiac ischemia and arterial thromboembolic events with the angiogenesis inhibitor bevacizumab in cancer patients: A meta-analysis of randomized controlled trials. Acta Oncol. 2010;49(3):287-297.

33. Pantaleo MA, Mandrioli A, Saponara M, et al. Development of coronary artery stenosis in a patient with metastatic renal cell carcinoma treated with sorafenib. BMC Cancer. 2012;12(1):231.

34. Sonpavde G, Bellmunt I, Schutz F, Choueiri TK. The Double Edged Sword of Bleeding and Clotting from VEGF Inhibition in Renal Cancer Patients. Curr Oncol Rep. 2012; 14(4):295-306.

35. Oi W-X, Shen Z, Tang L-N, Yao Y. Risk of arterial thromboembolic events with vascular endothelial growth factor receptor tyrosine kinase inhibitors: An up-to-date meta-analysis. Crit Rev Oncol Hematol. 2014;92(2):71-82

36. Proverbs-Singh T, Chiu SK, Liu Z, et al. Arterial thromboembolism in cancer patients treated with cisplatin: a systematic review and meta-analysis. J Natl Cancer Inst. 2012;104(23):1837-1840.

37. Schmidinger M, Zielinski CC, Vogl UM, et al. Cardiac toxicity of sunitinib and sorafenib in patients with metastatic renal cell carcinoma. J Clin Oncol. 2008; 26(32):5204-5212.

38. Khorana AA, Carrier M, Garcia DA, Lee AYY. Guidance for the prevention and treatment of cancer-associated venous thromboembolism. J Thromb Thrombolysis. 2016;41(1):81-91.

39. Anti-Platelet and Statin Therapy to Prevent Cancer-Associated Thrombosis. https://clinicaltrials.gov/ct2/show/NCT022 85738 [Last accessed 9 Oct 2017].

40. Lindhardsen J, Ahlehoff $\mathrm{O}$, Gislason GH, et al. Initiation and adherence to secondary prevention pharmacotherapy after myocardial infarction in patients with rheumatoid arthritis: a nationwide cohort study. Ann Rheum Dis. 2012;71(9):1496-1501.

41. Brown MT, Bussell JK. Medication Adherence: WHO Cares? Mayo Clin Proc. 2011;86(4):304-314

42. Scappaticci FA, Skillings JR, Holden SN, et al. Arterial thromboembolic events in patients with metastatic carcinoma treated with chemotherapy and bevacizumab. J Natl Cancer Inst. 2007:99(16):1232-1239.

43. Roe MT, Messenger JC, Weintraub WS, et al. Treatments, Trends, and Outcomes of Acute Myocardial Infarction and Percutaneous Coronary Intervention. J Am Coll Cardiol. 2010;56(4):254-263.

44. Grysiewicz RA, Thomas K, Pandey DK. Epidemiology of ischemic and hemorrhagic stroke: incidence, prevalence, mortality, and risk factors. Neurol Clin. 2008;26(4):871-895, vii.

45. Mellema WW, van der Hoek D, Postmus PE, Smit EF. Retrospective evaluation of thromboembolic events in patients with non-small cell lung cancer treated with platinum-based chemotherapy. Lung Cancer. 2014;86(1):73-77.

46. Reiner AS, Navi BB, DeAngelis LM Panageas KS. Increased risk of arterial thromboembolism in older men with breast cancer. Breast Cancer Res Treat. 2017;166(3):903-910.

47. Haguet $\mathrm{H}$, Douxfils J, Mullier F, Chatelain C, Graux C, Dogné JM. Risk of arterial and venous occlusive events in chronic myeloid leukemia patients treated with new generation BCR-ABL tyrosine kinase inhibitors: a systematic review and meta-analysis. Expert Opin Drug Saf. 2017;16(1):5-12.

48. Hultcrantz M, Pfeiffer RM, Björkholm M et al. Elevated risk of venous but not arterial thrombosis in Waldenström macroglobulinemia/lymphoplasmacytic lymphoma. Thromb Haemost. 2014;12(11):1816-1821. 